\title{
Short communication Decision making in local therapy for breast cancer
} Monica Morrow

Fox Chase Cancer Center, Surgical Oncology, Cottman Avenue, Philadelphia, Pennsylvania 19111, USA

Corresponding author: Monica Morrow, Monica.Morrow@fccc.edu

Published: 20 December 2007

This article is online at http://breast-cancer-research.com/content/9/S2/S8 (c) 2007 BioMed Central Ltd

Shared decision making has been accepted as a desirable approach to medical care, but our understanding of patient satisfaction with the process and its impact on treatment selection is limited. The local therapy of breast cancer is an ideal model for studying the decision-making process because there is a large body of high-quality evidence on which to base decisions. To study decision making, we used a population-based sample of all women with ductal carcinoma in situ (DCIS) and a 20\% sample of those with invasive breast cancer diagnosed in 2002 and reported to the Los Angeles and Detroit Surveillance, Epidemiology, and End Results registries. There was a $77 \%$ response rate to the survey, yielding 1,884 patients. More than 90\% were contacted within 6 months of diagnosis [1].

The mean patient age was 60 years and $70 \%$ were white. Less than a high school level of education was reported by $27 \% ; 61 \%$ graduated from high school or had some college education, and 13\% were college graduates. The mastectomy rate was $30 \%$ for patients with both DCIS and invasive cancer. Accepted clinical contraindications to breast-conserving surgery were reported by $11.5 \%$ of the study population. In $41 \%$ of cases the patient reported that she was the primary decision maker, the decision was shared in $37 \%$, and was made by the surgeon in $22 \%$ of cases. The therapy recommended by the surgeon was breast-conserving surgery in $49 \%$, mastectomy in $15 \%$, and patients reported being offered a choice between the two procedures in $37 \%$ of cases. Greater patient involvement in the decision-making process was significantly correlated with treatment by mastectomy after adjustment for multiple clinical and demographic variables. Only $5.8 \%$ of women whose surgeon made the treatment decision had a mastectomy, as compared with $16.8 \%$ who reported a shared decision and $27 \%$ of women who reported that they made the decision $(P=0.003)$.

Concern about disease recurrence was the most influential factor in treatment choice, with $40 \%$ of women reporting that their treatment choice was greatly influenced by this concern. Concerns about disease recurrence were strongly associated
Breast Cancer Research 2007, 9(Suppl 2):S8 (doi:10.1186/bcr1806)

with receipt of mastectomy; $52 \%$ of women who were greatly concerned about disease recurrence received a mastectomy, as compared with $19 \%$ of those who were not influenced, or only slightly influenced, by this concern $(P<0.001)$ [1].

Patients expressed a high level of confidence in decision making, with more than $80 \%$ of women of all ages being very or extremely confident about their treatment choice. However, fewer than $50 \%$ were able to answer correctly a true-false question about the lack of a survival difference after treatment by mastectomy or lumpectomy and radiation.

We also examined the match between decision control and patient preference in a subset of 1,028 patients with no contraindications to breast-conserving therapy who were treated by 270 surgeons [2]. We found a rate of mismatch between desired and actual level of involvement of 31\%. In $20 \%$ of cases actual involvement in decision making was greater than preferred, whereas in $11 \%$ it was less than preferred. Twenty per cent of patients reported that they asked for but did not receive a treatment recommendation, and $16 \%$ noted that they did not ask for but received a treatment recommendation. Patients who perceived too little involvement in the decision-making process were younger, reported that only one treatment option (mastectomy or breast conservation) was discussed, and were more likely to have been seen by a high-volume surgeon. Patients who perceived too much involvement in decision making had lower levels of education and felt that they asked for but did not receive a treatment recommendation. Lack of satisfaction with the decision-making process was expressed by 37\%, lack of satisfaction with the relationship with the surgeon by $23 \%$, and lack of satisfaction with communication with the surgeon by $24 \%$ of patients [3]. Satisfaction with the decision-making process did not correlate with surgeon sex, years in practice, or treatment in a cancer centre. Increased satisfaction with decision making was significantly associated with high-volume breast surgeons, and a trend toward increased satisfaction with higher breast surgery volume was observed for other aspects of the treatment selection process. 
The results of these studies suggest that although patients believe that they are informed about their treatment choices and are confident in their decisions, major knowledge gaps exist. In addition, for a significant number of patients there is a mismatch between the preferred level of involvement in decision making and what actually occurs. As in the treatment of breast cancer, the style of decision making must be tailored to the individual.

\section{Acknowledgement}

This article has been published as part of Breast Cancer Research Volume 9 Supplement 2, 2007: Controversies in Breast Cancer. The full contents of the supplement are available online at http://breastcancer-research.com/supplements/9/S2.

\section{References}

1. Katz SJ, Lantz PM, Janz NK, Fagerlin A, Schwartz K, Liu L, Deapen $D$, Salem B, Lakhani I, Morrow M: Patient involvement in surgery treatment decisions for breast cancer. $J$ Clin Oncol 2005, 23:5526-5533.

2. Hawley ST, Lantz PM, Janz NK, Salem B, Morrow M, Schwartz K, Liu L, Katz SJ: Factors associated with patient involvement in surgical treatment decision making for breast cancer. Patient Educ Couns 2007, 65:387-395.

3. Waljee JF, Hawley S, Alderman AK, Morrow M, Katz SJ: Patient satisfaction with treatment of breast cancer: does surgeon specialization matter? J Clin Oncol 2007, 25:3694-3698. 